\title{
Income Tax for Employees using Visual Basic and Access Database
}

\author{
Ruba T. Ibrahim \\ Mohamed I. Mohamed \\ Nashwan A. Ramadan \\ rubatalal83@gmail.com \\ nashwann179@gmail.com \\ College of Computer Sciences and Mathematics \\ University of Mosul
}

\section{Received on: 31/12/2018}

Accepted on: 24/01/2019

\begin{abstract}
In previous years, database applications were built in the form of a file system, but many disadvantages were found after that, such as: data replication, inconsistency while storing, difficulty in accessing data and need to write a new program to execute each new task in addition to isolating the data within multiple file formats ..... etc. All the above problems were solved by using database systems.

This paper is an applied to demonstrate the effective role of the tax information system to establish and operate knowledge database as a knowledge management requirement by: the conceptualization of the income tax, knowledge management system and the development of the retirement information system.

In this paper, the ACCESS database was created and linked with the Visual Basic language to calculate income tax of the employees. Where the information of employee is entered (such as the employee's name, number and social status as well as the nominal salary). The system has saved and modified information of employees , printing a report on one employee or more, as well as knowing the amount of tax that got from employees.
\end{abstract}

Keywords: Database, tax information system, knowledge management, employee records, Visual Basic, Access database.

$$
\begin{aligned}
& \text { برنامج حساب ضربية الدخل للموظفين باستخدام الفيجوال بيسك وقاعدة البيانات الاكس }
\end{aligned}
$$

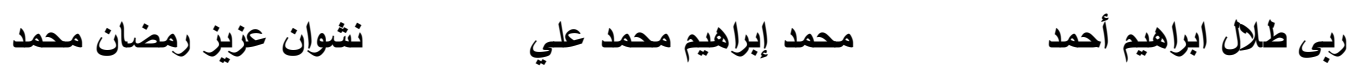

$$
\begin{aligned}
& \text { كلية علوم الحاسوب والرياضيات } \\
& \text { جامعة الدوصل، الموصل، العراق }
\end{aligned}
$$

تاريخ قبول البحث: 2019101124

تاريخ استلام البحث: 2018\12131

\section{الملخص}

بنيت في السنوات السابقة تطبيقات قواعد البيانات على شكل نظام ملفات(File System). لكن سرعان ما

ظهرت عيوب كثيرة بعد استخدامها مثل :تكرار البيانات وتضاربها عند الخزن ،وتكرار البيانات في أكثر من ملف لف لفي

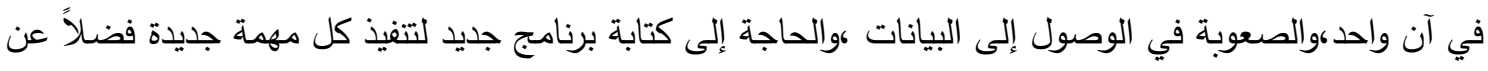
عزل البيانات ضمن ملفات متعددة الصيخ.....الخ • حلت المشاكل المذكوره أعلاه باستخدام أنظمة قواعد البيانات. 
يعد هذا المشروع دراسة تطبيقية ونظرية لبيان الدور الفعال والمؤثر لنظام المعلومات الضريبي في إنثاء

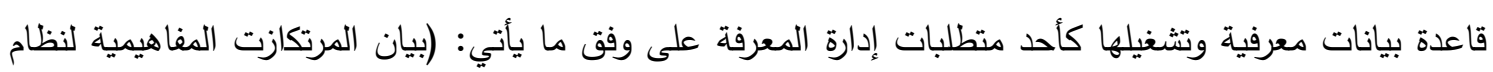
ضريبة الدخل ولإدارة المعرفة، وتطوير نظام المعلومات المتعلق بالتقاعد) . عمل في هذا المشروع قاعدة بيانات الاكسس وربطها بلغة الفيجوال بيسك لبيان حساب ضرئ ضريبة الدخل

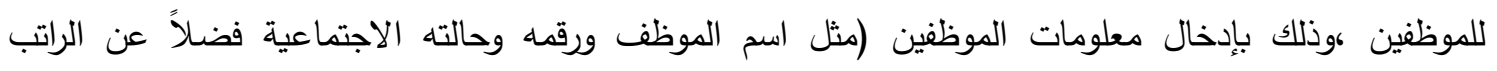
الاسمي)، يقوم النظام بحفظ معلومات كبيرة عن الموظفين وإمكانية التعديل ،والخزن ،والإضافة عليها وحذفها عليها وسهولة البحث ومعرفة عدد سجلات الموظفين ،فضلاً عن طباعة تقرير موظف واحد أو مجموعة من تقارير الموظفين فضلاً عن معرفة كمية الضريبة المستحصلة من الموظفين. الكلمات المفتاحية: قاعدة بيانات، نظام معلومات ضريبي، إدارة المعرفة، سجلات الموظفين، فيجوال بيسك، قاعدة البيانات الأكسس.

\section{1. المقدمة Introduction}

الضريبة هي اقتطاع مالي يدفعه الأفراد إلى الدولة بطريقة إلزامية مساهمة منهم في التكاليف والاعبـاء العامة

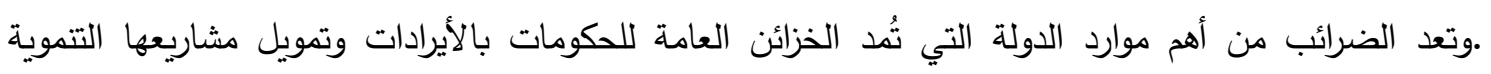
الحضارية[1]،توجد أنواع عديدة من الضرائب ، لكن في هذا البحث تم التركيز على المقدار الضريبي في رواتب الموظفين فقط . ونظراً لأهمية النظام الضريبي تطرق البحث إلى الأساليب والطرائق الفعالة لدعم هذا النظام وتتميته، ويعد النظام الضريبي اليوم وسيلة فعالة يهدف أساساً إلى مساعدة الإدارة ومصالح الضرائب في إثبات ذاتها لتوفير الموارد

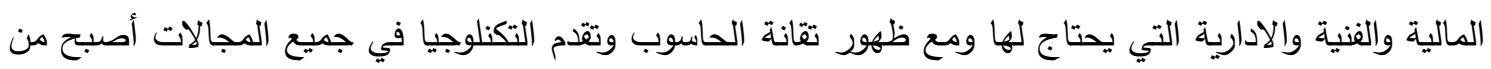
الضروري أن يكون لهذه التقانة أثر واضح في دفع الحصيلة الضريبية لإنجاح أهداف النظام الضريبي من جهة

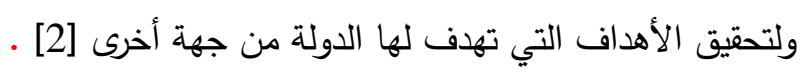
لذلك عمل في هذا البحث قاعدة بيانات الاكسس وربطها بلغة الفيجوال بيسك لبيان حساب ضريبة الدخل للموظفين و ذلك بإخال معلومات الموظفين (مثل اسم الموظف ورقمه وحالته الاجتماعية فضلاً عن الراتب الاسمي)،يقوم النظام بحفظ معلومات كبيرة عن الموظفين وامكانية التعديل ،والخزن ،والإضافة عليها وحذفها وسهولة البحث ومعرفة عدد سجلات الموظفين ،فضلاً عن إمكانية طباعة تقرير موظف واحد أو مجموعة من تقارير الموظفين فضلاً عن معرفة كمية الضريبة المستحصلة من الموظفين . تفرض الضريبة على كل الأشخاص الذين يتقاضون مرتبات وأجور حسب قانون ضريبة الدخل رقم 113 لسنة

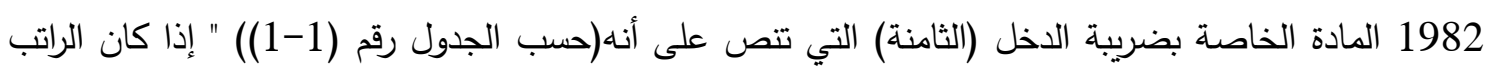
الاسمي أكثر من مليون دينار فسوف يضاف إلى السطر الأول العمود (د) ،ثم نطرح السطر الثاني من السطر لثربه

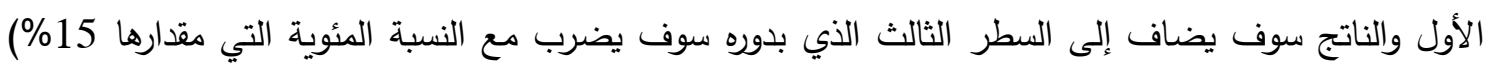

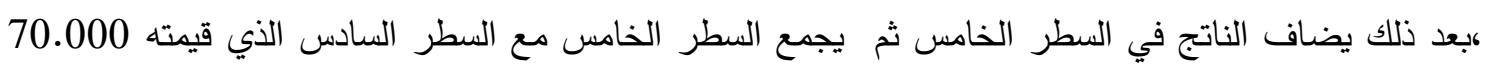

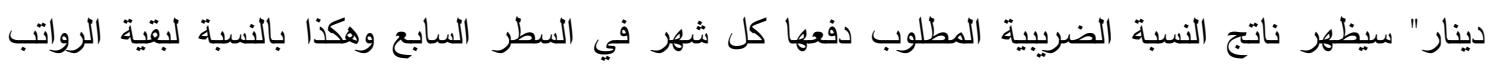

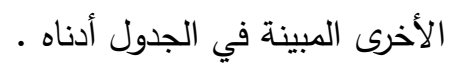

إذا كان الاخل الخاضع للضريبة : الاخينه 
•ا يتجاوز 250,000 دينار فأدخله في السطر 1 من العمود أ من الجدول أدناه .

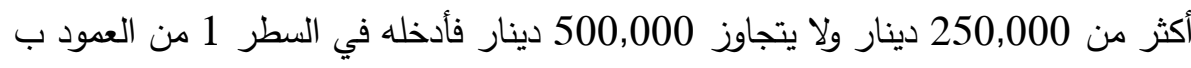

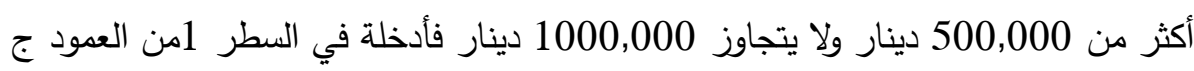

أكثر من 1000,000 دينار فأدخلة في السطر 1 من العمود د ـ داتجاوز

الجدول رقم (1-1):يبين قانون ضريبة الدخل رقم 113 لسنة 1982 المادة(الثامنة)

\begin{tabular}{|c|c|c|c|c|}
\hline \multicolumn{2}{|r|}{ أ } & ب & ج & د \\
\hline 1 & صفر دينار & 250.000دينار & 500.000 دينار & 1000.000 دينار \\
\hline 2 & & & & \\
\hline 3 & & & & \\
\hline 4 & $\% 3$ & $\% 5$ & $\% 10$ & $\% 15$ \\
\hline 5 & & & & \\
\hline 6 & صفر دينار & 7,500 دينار & 20,000 دينار & 70,000 دينار \\
\hline 7 & & & & \\
\hline
\end{tabular}

2.1 2. - 2. الدراسات والأعمال السابقة

بدأت معظم الثركات التجارية خاصة في البلدان المتقدمة بتخزين ملفاتها وحفظها على الكمبيوتر منذ عام 131960] إذ كان مسؤول أي شركة يقوم بالاحتفاظ أو استرجاع البيانات اللازمة عن طريق استخدام نظام الملفات

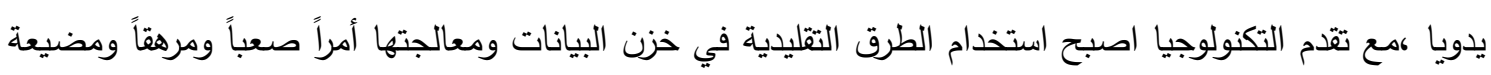

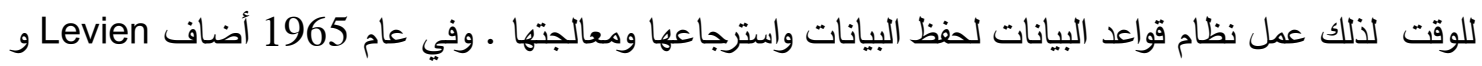

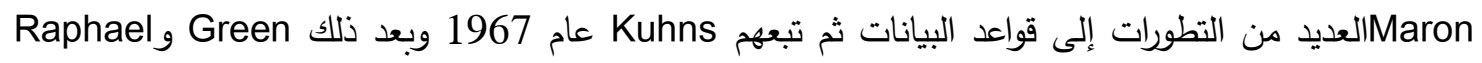

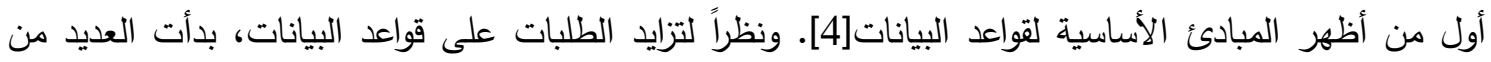

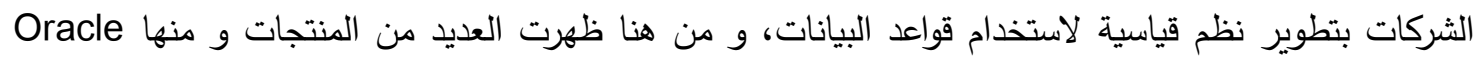

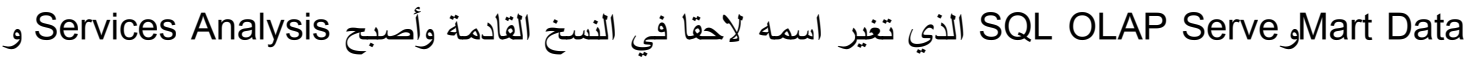
بعض المنتجات الأخرى.إذ كانت تكلفة شراء هذه الأنظمة عالية نسبياً، فقد تصل إلى خمسة وعشرين ألف دولار

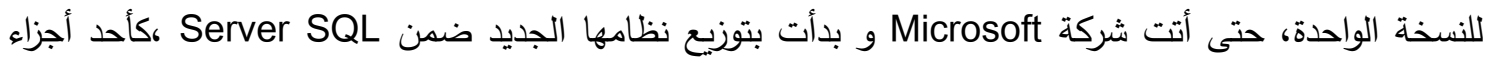

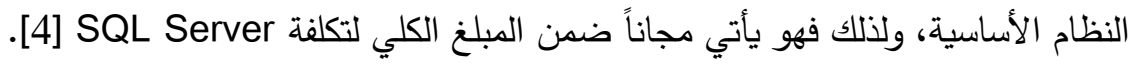

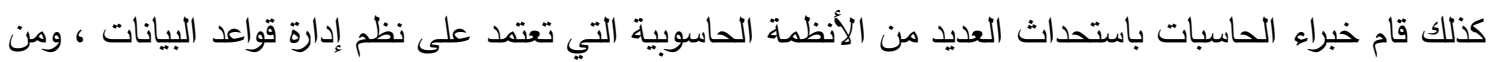
هذه الأنظمة: نظام حجز الطيران ،إذ يحتوي هذا النظام معلومات عن حجز مقعد مع تحديد الوجبة المفضلة للعميل

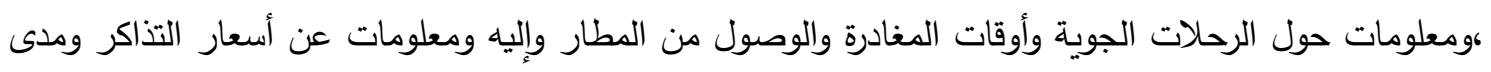

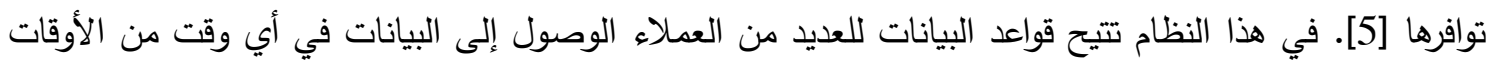

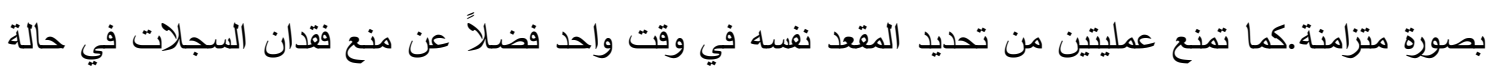

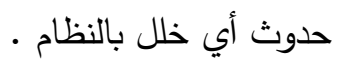


أما النظام الآخر فهو نظام البنوك ،وتثمل بياناته أسماء وعناوين العملاء والحسابات والقروض والأرصدة الخاصة بهم، فضلاً عن العلاقة بين العملاء وحساباتهم والقروض العائدة لهم ،إذ يقوم العميل بالاستعلام وتحديث بياناته في ولاتي آن واحد عبر الصراف الآلي (ATM) أو عبر الانترنت كما هو الحال مع نظام حجز الطيران ،إنَ نظام البنوك بكان الجديد يوفر الوصول المتزامن للبيانات دون حدوث فقدان لها. على سبيل المثال، عند إخراج المال من جهاز الصراف الآلي(ATM)، يجب على البنك أن يسجل المبلغ المسحوب، حتى في حالة حدوث عطلٍ ما، ومن ناحية

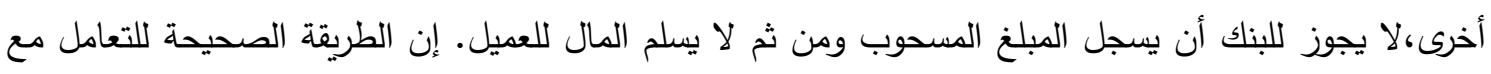

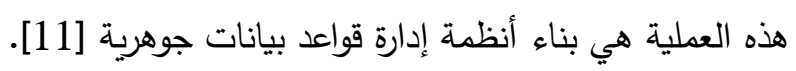
يوجد نظام آخر الذي يتعلق بعمل سجلات للشركات التي تتضمن معلومات عن المبيعات ،

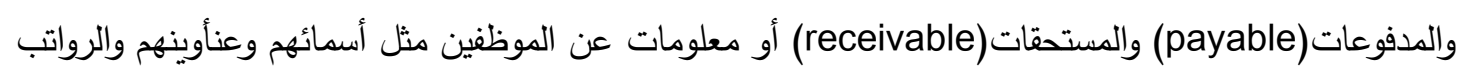
المدفوعة لهم فضلاً عن عمل قاعدة بيانات لتحديد الدخل الضريبي المفروض على رواتبهم ، ومن ثم عمل تقارير

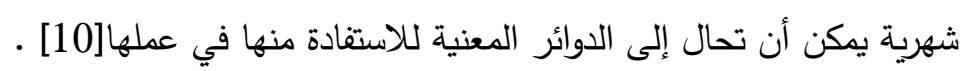
3.1. 3قــــمة عن قواعد البيانات

اصبحت البيانات مع تقدم التكنولوجيا صغيرة ومترابطة نسبياً فضلاً عن زيادة متطلبات مسؤولي الثركات

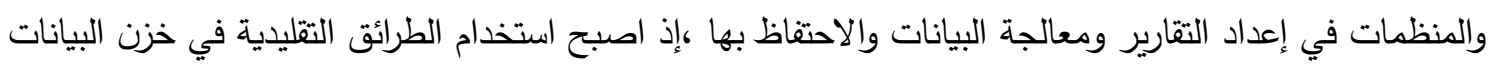
ومعالجتها أمراً صعباً ومرهقاً ومضيعة للوقت .مما دفع العلماء إلى عمل ما يسمى بقواعد البيانات (Data Base) والتي تمثل مجموعة كبيرة ومتحدة من البيانات وهي بحد ذاتها مجموعة من الحقائق والأرقام التي يتم معالجتها

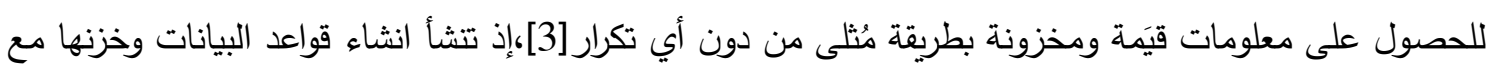

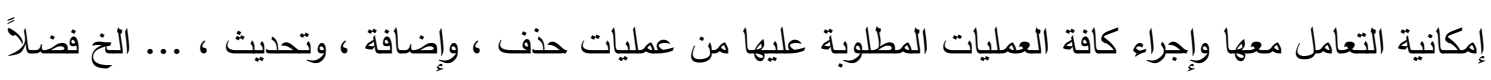
عن توفير طرائق مختلفة مثل (الثاشة أو الطابعة) لعرض البيانات في قاعدة البيانات ، وذلك باستخدام نظام إدارة

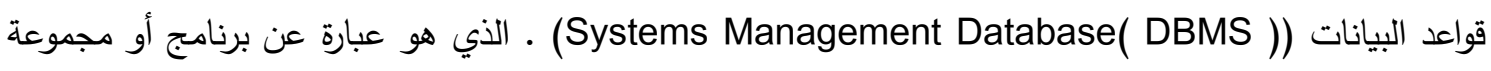

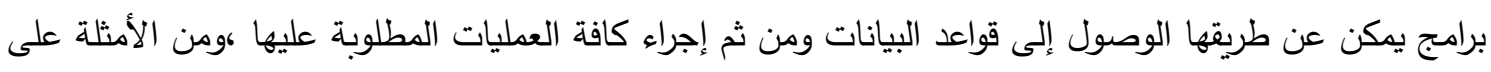
قواعد البيانات : دليل الهاتف ،و نظام Rolodex file وفهرس البطاقات (card catalog) في المكتبات العلمية المحلية ..الخ الخ الخ

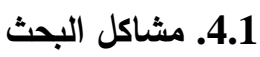

عدم تطبيق نظم المعلومات الحديثة والاعتماد على الاساليب اليدوية في العمل الضريبي جعل هناك صعوبة في تقديم المعلومات في الوقت المناسب وبأقل جهات وتكلفة.

صعوبة تقديم المعلومات في الوقت المناسب يؤدي إلى عدم كفاءة الإدارة ،فضلاً عن إمكانية إدخال

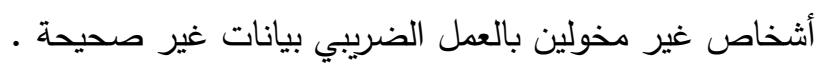

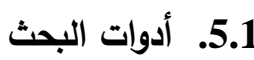

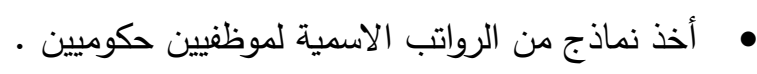

استخدام برنامج مأيكروسوفت اكس (2013)(Microsoft Access) • 
• استخدام برنامج فجول بيسك (Visual Basic) الاصدار السادس في تصميم القاعدة المعرفية

والواجهات الاساسية للنظام الضريبي.

اعتماد الأنموذج العلاقاتي بربط جداول قاعدة البيانات المعرفية .

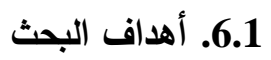

1. حوسبة العمل الضريبي ، ورفع مستوى المعلومات ودقتها وسرعتها وتتظيمها وتطوير مستوى الضیى

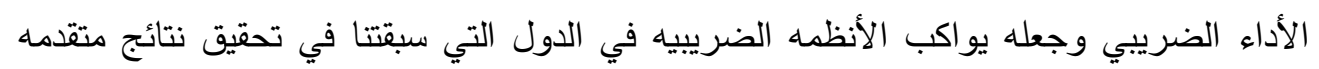

\section{لمجتمعاتها .}

2. تصميم قاعدة البيانات باستخدام Microsoft Access و و ون ثن ثن استخراج التقارير

من قاعدة البيانات ـ كذلك هدفت هذه الدراسة إلى تقليل الوقت والجهة وزيادة سرعة انجاز الأعمال .

\section{1. - المنهجية العملية للبحث}

1.7.1.واجهة تنفيذ اليرنامج

عند تتفيذ اليرنامج تظهر لاينا واجهة الحمأيه أو الأمنية للإنامج، وذلك لغرض حمأية البيانات ومنع وصول

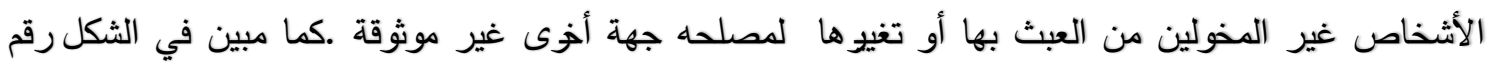

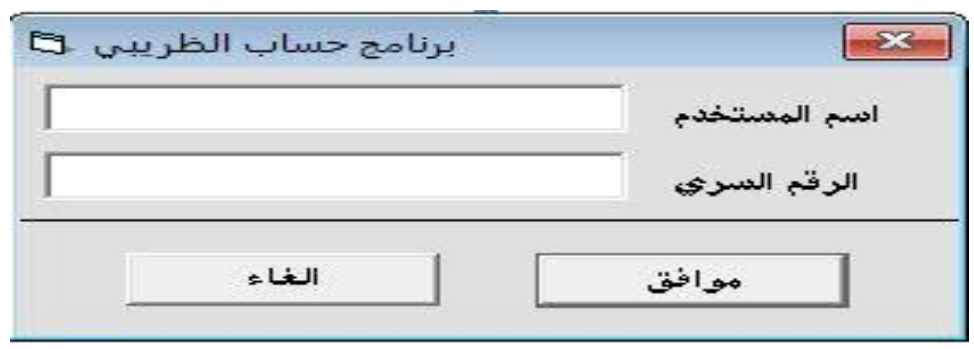

$$
\text { الثكلرقم (1-1)واجهة الامنية لللونامج }
$$

2.7.1

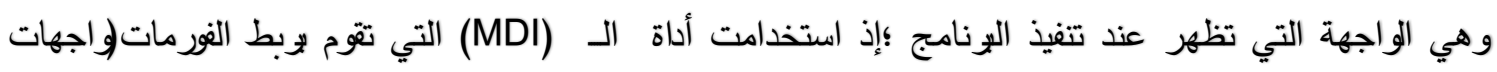
الئنامج الأخىى)مع بعضها البعض، كذلك منها نختار بقية الواجهات الأخىى للاخول اليهم وتتفيذ عمليات الإضافة ، والبحث و التعديل أو طباعة تقير الخ ..الثكل رقم (1-2) يوضح الواجهة الوئيسة للرنامج. ومن خصائص

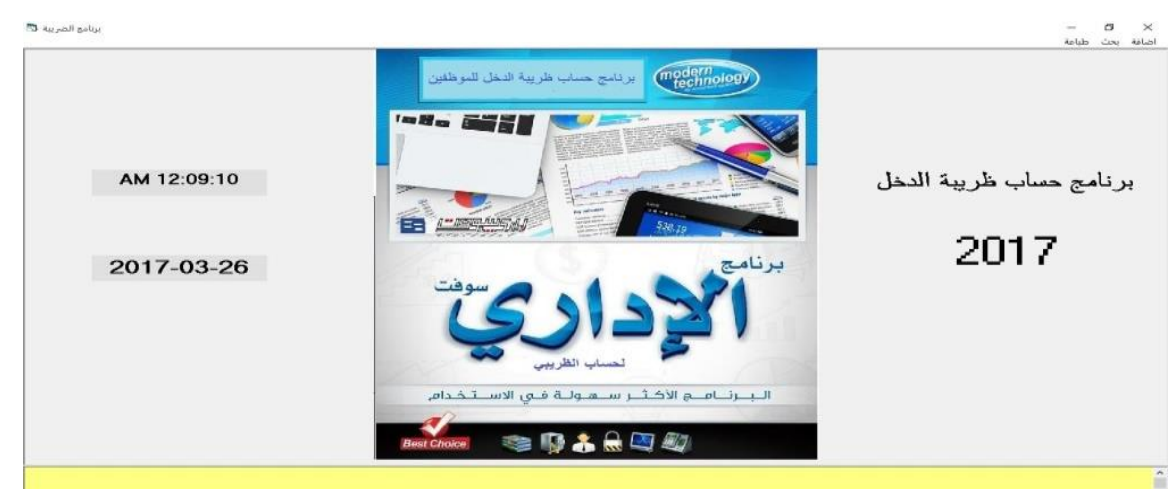


هذه الواجهة عند اختيار إحدى الواجهات مثلاً الإضافة ،فإنه لأيمكن استخدام بقية الواجهات في الوقت نفسه وذلك لتحقيق الأمنية والحفاظ على سلامة البيانات . الثكلرقم (1-2):الواجهة الأئيسة للإنامج

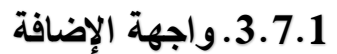

في هذه الواجهة كما موضح في الثكل رقم (1-3) فإنها تحتوي على رقم الموظف(ID) لتمييز كل موظف عن الآخر • فإذا كانت الأسماء متشابهة في بعض الحالات، فيكون رقم الموظف هورقم خاص يمزه عن غره وليس له علاقة بتسلسل الموظف. كذلك تحتوي على حقل اسم الموظف والواتب الاسمي وحالة الفد إذا كان (متروجًا لُوغير

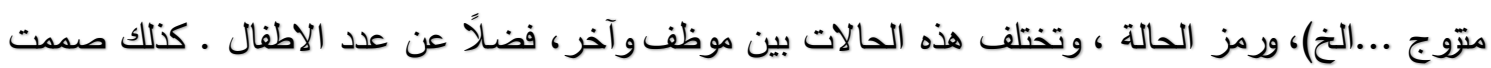
واجهة الإضافة بحيث تظهر في وسط شاشة الحاسوب ، وذلك لجعل واجهات اللرنامج بالحجم والتنسيق نفسه فضلًا عن ذلك ، يقوم الليانامج بتهيئة قاعدة البيانات حتى إذا كانت مخزونة في مسار آخر غير المسار المخزون.

$$
\text { فيه الليرنامج كما ملرمج في الكود الآتي : }
$$

If $d b$. State $=$ adSateOpen Then $d b$. Close

$\mathrm{db}$. Provider $="$ Microsoft . JET . OLEDB . 4 . $0 "$

$\mathrm{db}$. Open App . Path \& "\dareba . mdb"

IF rs . State $=$ adStateOpen Then $r$. Close

rs . open " [dareba ]" , db , adOpenDynamic , adLocPessimistic

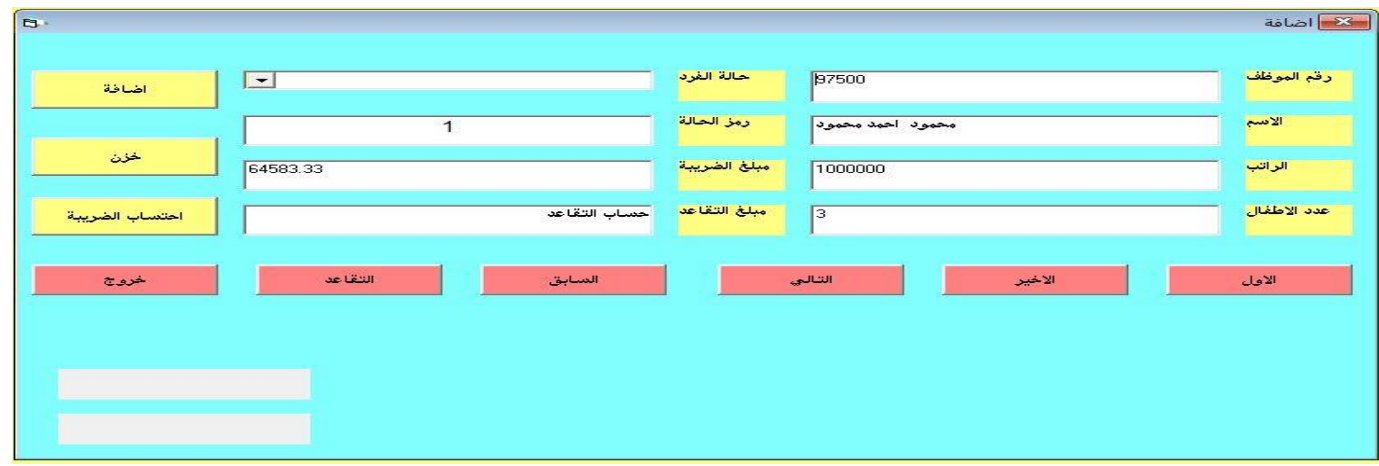

الثكل رقم (1-3)واجهة الإضافة

يتم احتساب الضريبة لكل موظف من خلال القانون الضريبي المأخوذوالمعتمد من قبل وزلة المالية الواقية .

ويعتمد أيضا على قيمة الإتب وحالة الفود، إذ تم برمجة عملية الاحتساب الضريبي حسب الكود أدناه :

$\mathrm{X} 1(1)=4500000 \quad \mathrm{X} 1(5)=2500000$

$\mathrm{X} 1(2)=3100000 \quad \mathrm{X} 1(6)=4500000$

$X 1(3)=4500000 \quad X 1(7)=2500000$

$\mathrm{X} 1(8)=5000000$

$\mathrm{X} 1(9)=5000000$ 
$\mathrm{X} 1(4)=2500000$

$\mathrm{Sq}=$ " select $*$ from dareba order by name "

rs . open sq 11 , can , adOpenDynamic, adLockPessimistic

swifts $=$ Text5 . Text

Whiled $=$ Text 3 . Text

Salary $=$ Text 2 . Text

nnst $=$ salary $* 12-0.1 *($ salary $* 12)$

At $a x=$ nnst $-(x 1$ (swifts $)+($ whiled $* 200000))$

• زر الإضافة

عند الكبس على زر الإضافة سوف يقوم بتهيئة سجل فل غاخل داخل قاعدة البيانات وذلك لإضافة بيانات موظف جديد

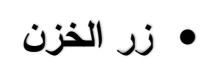

عند الكبس على زر التزن سوف يقوم بفحص الحقول إذا كانت فلغة فسوف تظهر رسالة تحذيرية بعدم وجود بيانات لغرض ترنها ـ أما في حالة إدخال البيانات والكبس على زر التزن سوف تخزن البيانات داخل قاعدة

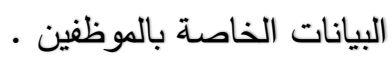

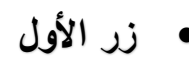

عند الكبس على زر (الأول) يتم الذهاب إلى العنصر الأول في قاعدة البيانات . •

عند الكبس على زر (التالي) يتم الذهاب إلى سجل الموظف الذي يليه .أما في حالة كنا في السجل الأخير سوف

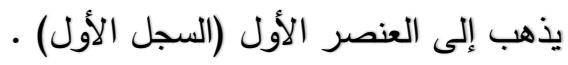

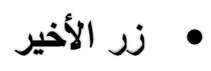

عند الكبس على زر (الأخير) يتم الذهاب إلى العنصر الأخير (السجل الأخير) في قاعدة البيانات . زر السابق

عند الكبس على زر (السابق) يتم الذهاب إلى سجل الموظف الذي يسبقه .أما في حالة كنا في السجل الأول فسوف يذهب إلى العنصر الأخير (السجل الأخير) .

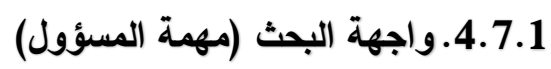

تعد هذه الواجهة من أهم الواجهات في الليرنامج ،لأنها تحقي على قاعدة البيانات الخاصة بالموظفين فضلًا

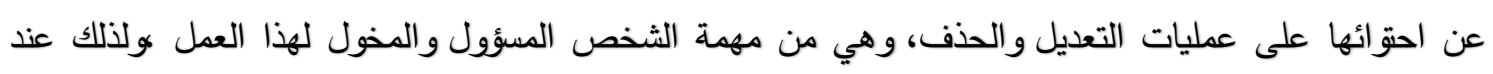

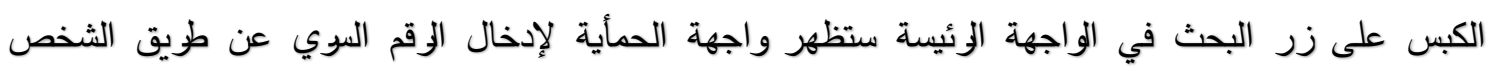
المخول(المؤول) وذلك لحماية البيانات من الأشخاص المتطفلين •

استدعيت في هذه الواجهة قاعدة البيانات المخزونة في الاكسس وعرضت عن طويق الأداة (GridData)

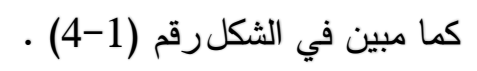


إذ يبحث عن أي موظف بكتابة الاسم كاملًا أو خذء من الاسم ، وذلك لتحقيق البرعة في البحث ووض ف أكثر من اسم (سجل)، يتضمن العرف نفسه العرف معالجة حالة نسيان الاسم أو إدخال خذء من الاسم ،ثم يعرض الأسماء التي لها علاقة بالرف المدخل داخل الأداة (GridData) ) ثم نقوم بالضغط(Doubleclick) على الاسم الكواد البحث عنه ستظهر المعلومات الخاصة به داخل أبوات (Texts) نقوم بتعديل بياناته مثل( الحالة الاجتماعية أو زيادة عدد الأطفال) هوتتحدث هذه المعلومات بمدد متعاقبه

مثلًا كل شهر أو كل سنه على وفق الحالات لدينا ، ما عدازيل زر رقم الموظف؛ لأنه ثابت لا يتغير (ID). وعند الكبس على زر التعديل يظهر لدينا زر الحفظ التي كانت مخفية (غير مفعلة)وبعد تعديل البيانات نضغط على زر الحفظ لغض حفظ البيانات بعد تعديلها .

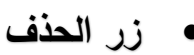

عند الكبس على هذا الزر تحذف بيانات الموظف (السجل) المؤشر عليه في قاعدة البيانات أو المعروضة بياناته في (Texts) كود الحذف :

Private sub command8_click()

\section{Adodc1.Recordset.Delete}

End sub

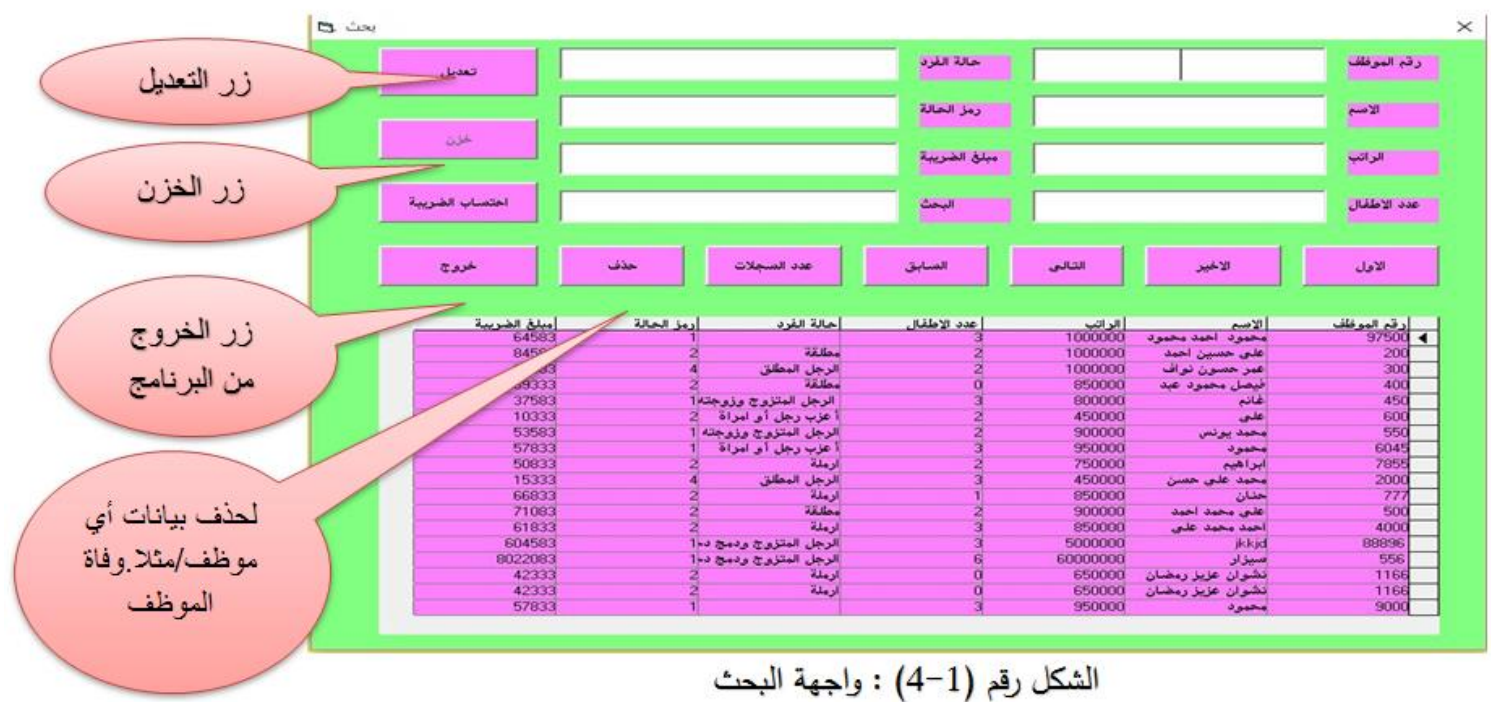

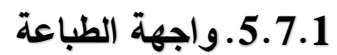

تحتوي واجهة الطباعة على قاعدة بيانات الموظفين و زر طباعة الفود (موظف واحد)فضلًا عن طباعة عامة لمجوعة تقلزير الموظفين ، ؤلًا: يتم البحث عن اسم الموظف ضمن قاعدة البيانات كما وضحناها سابقًا في واجهة البحث ، ثانيًا: عند الكبس على زر طباعة الفدد سوف تعرض بيانات الموظف فضلًا عن عرض طريقة 
حساب ضريبة الدخل المستقطعة يويا .ثالثا :عند الكبس على زر التقير العام سوف تعوض بيانات الموظفين الموجودة في قاعدة البيانات فضلًً عن حساب مجوع الضرائب المستحصلة من الموظفين . ونلاحظ في هذه الواجهة عدم إمكانية تعديل البيانات نهائيًا؛ إذ تستخدم لغزض العرض والطباعة فقط

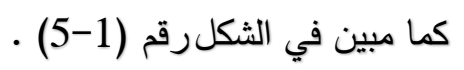

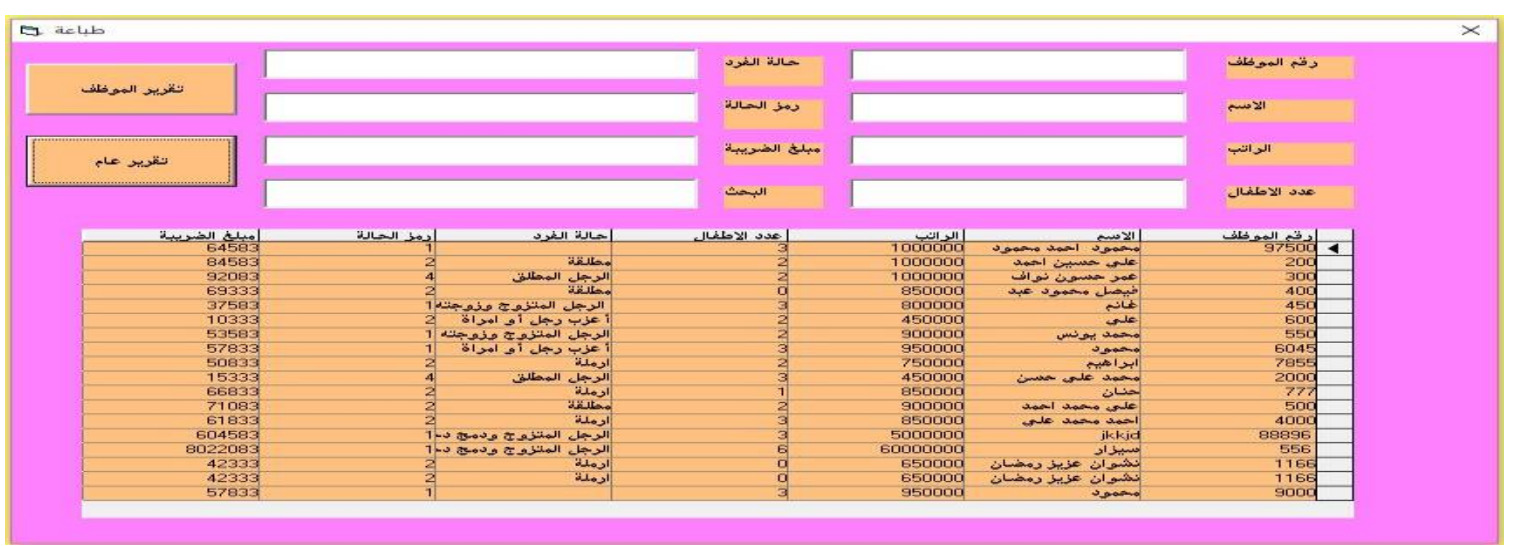

الثكلرقم (1-5) ؤاجهة الطباعة

\subsection{1. خطوات عملية انشاء تقرير}

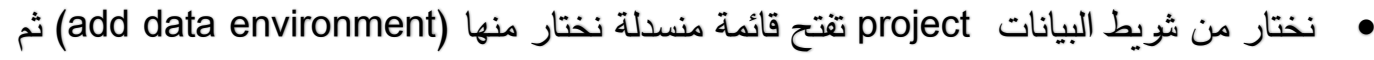
تظهر قائمة أخىى فنكبس (RightClick) على الـ communication ثم نختار properties كما

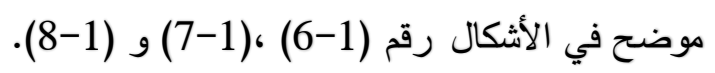

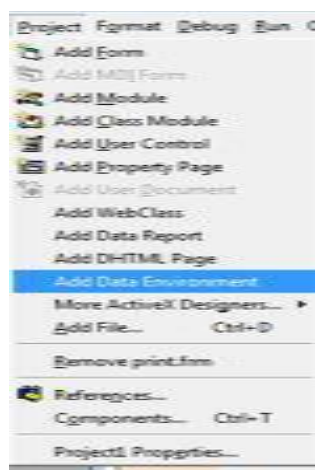

الثكل رقم (1-6):عملية انثاء تقرير

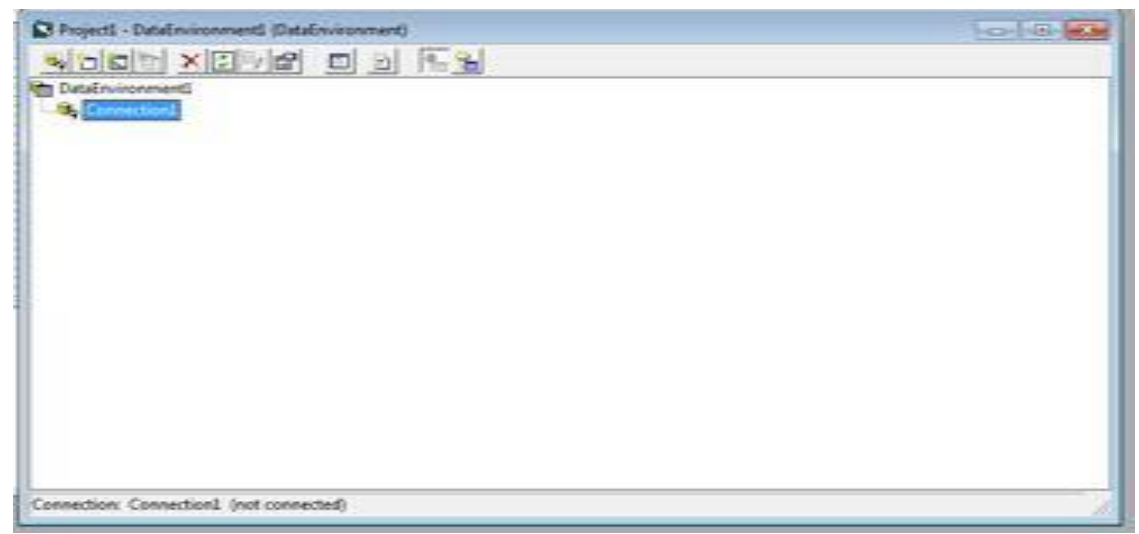


الثكل رقم (1-7):عملية انثاء تقرير

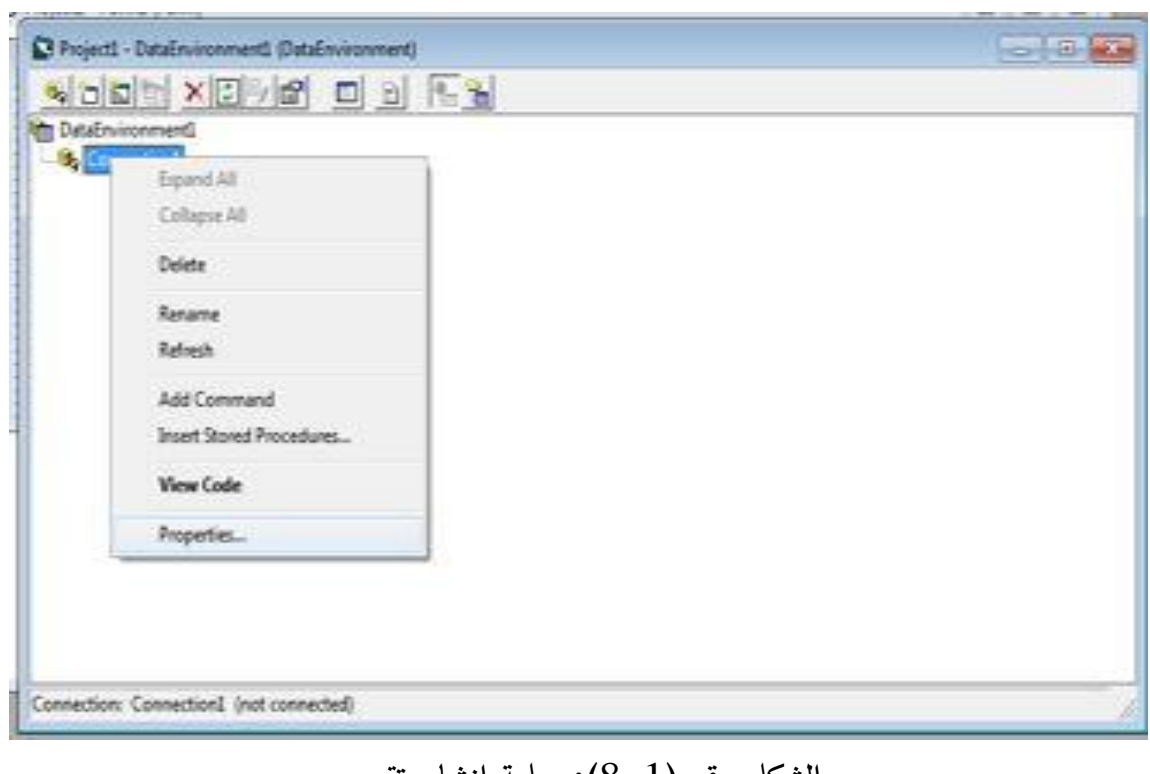

الثكل رقم (1-8):عملية انشاء تقرير

ثم بعد ذلك تظهر نافذة data link ونختار (Microsoft jet 4.0 OLE provider) ثم next كما

موضح بالشكل رقم (1-9).

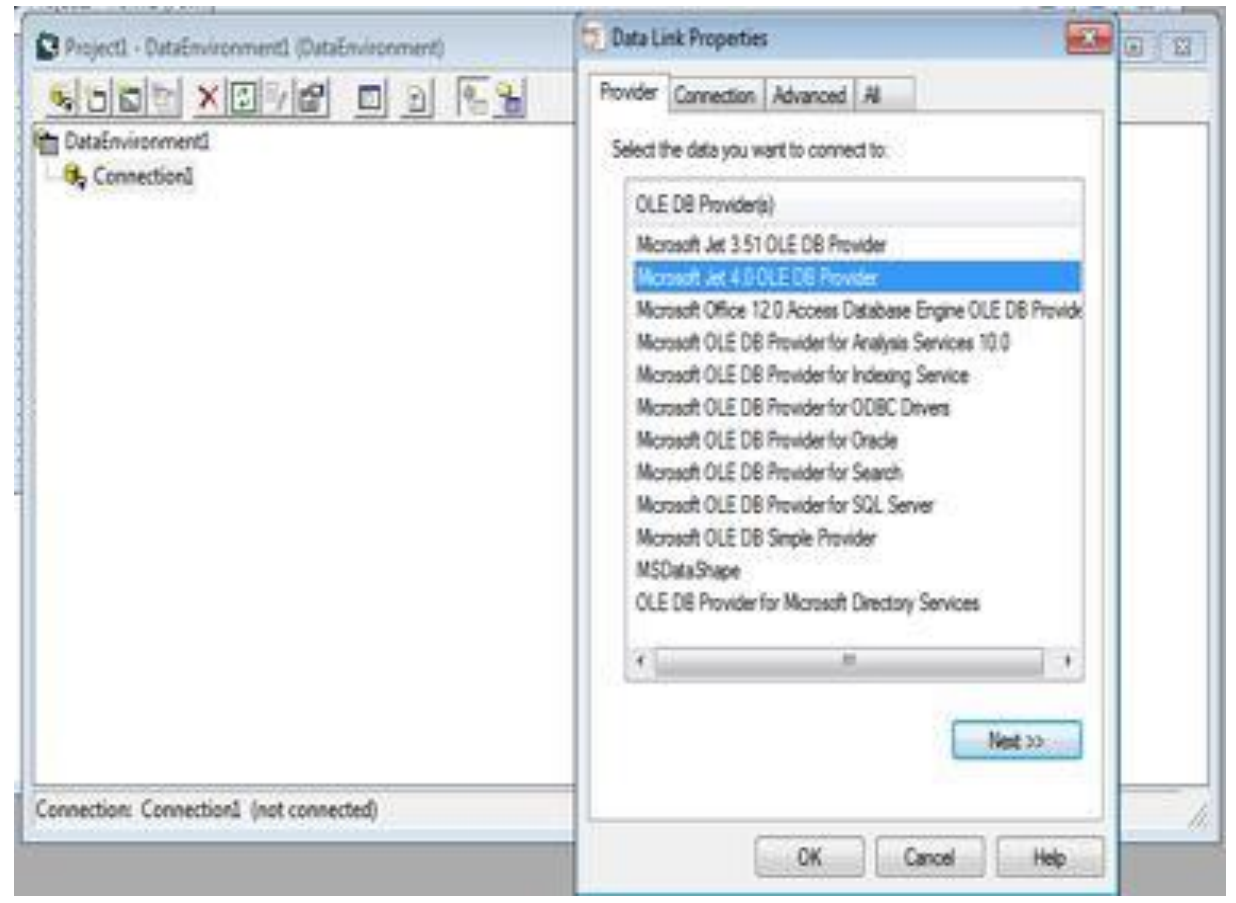

Microsoft jet 4.0 OLE provider الثكل رقم (1-9):عملية اختيار

$$
\begin{aligned}
& \text { • تقتح نافذه أخىى نختار connection وفي المستطيل رقم واحد } \\
& \text { • ثما مبين في الثكل رقم (1-10). }
\end{aligned}
$$




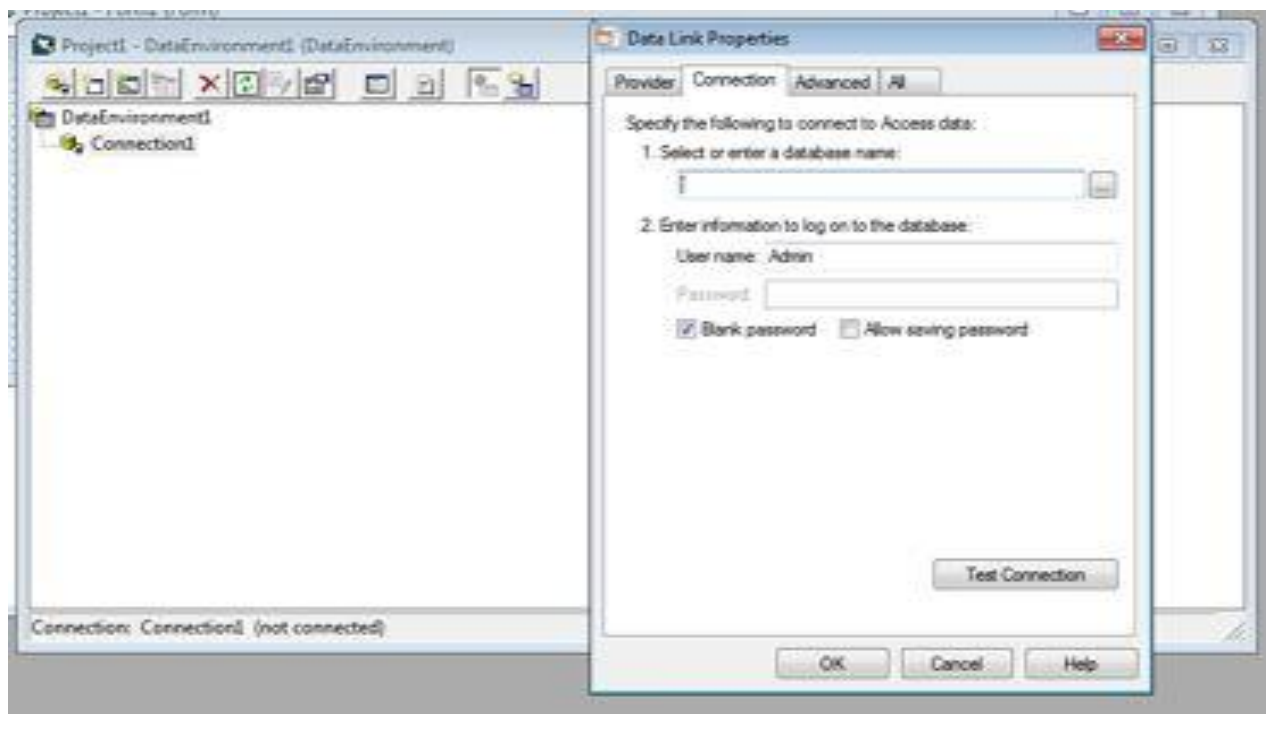

الثكل رقم (1-10):عملية الارتباط

ثم موى أخى من project نختار ( Add Data Report ) كما مبين في الثكلرقـم (1-11).

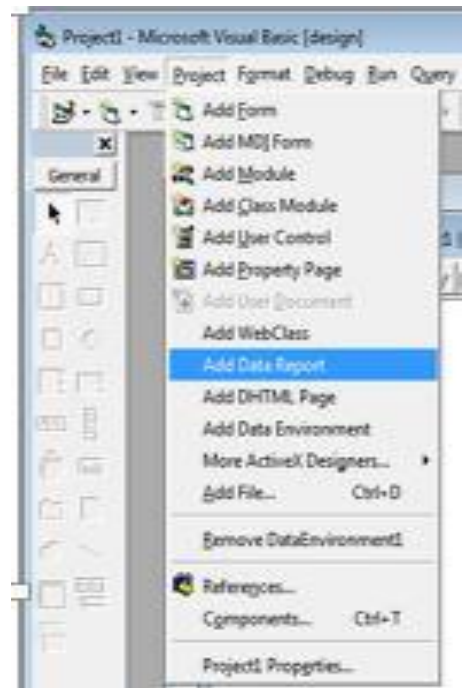

الشكلرقم (1-11):عملية اختيار (Add Data Report)

• ثم نكبس (RightClick) على communication و نختار Add Command كما في الثكلرقم 


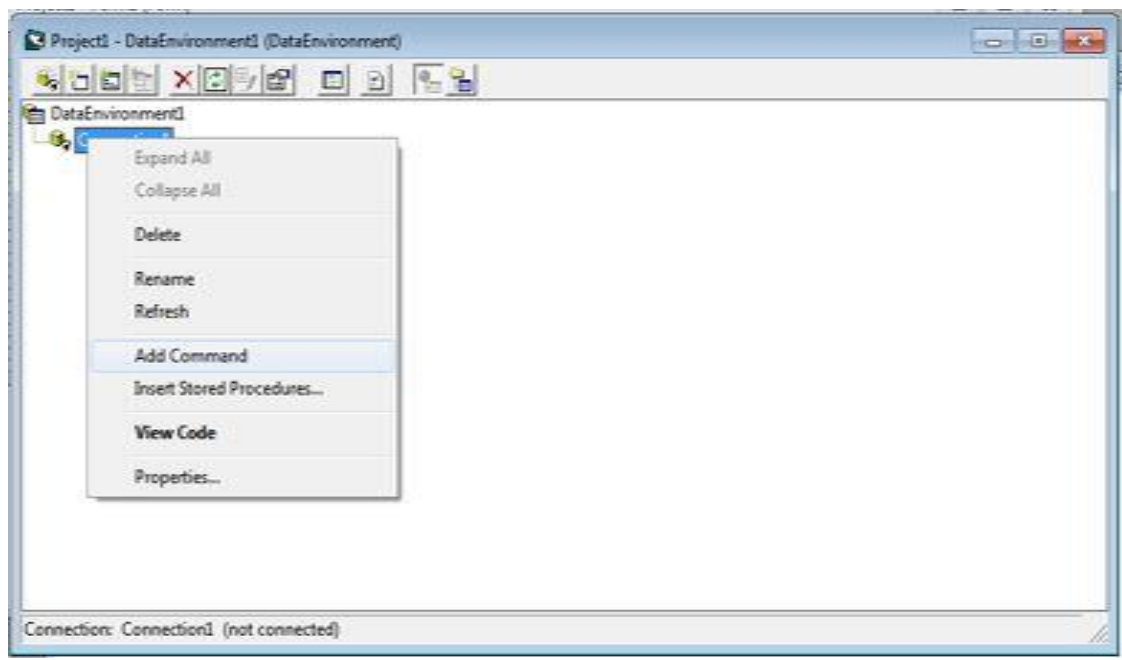

الثكل رقم (12-12):عملية اختيار (Add Command)

من Command نعمل (RightClick) ونختار properties كما موضح في الأشكال رقم (-13

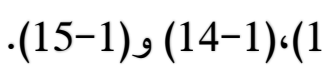

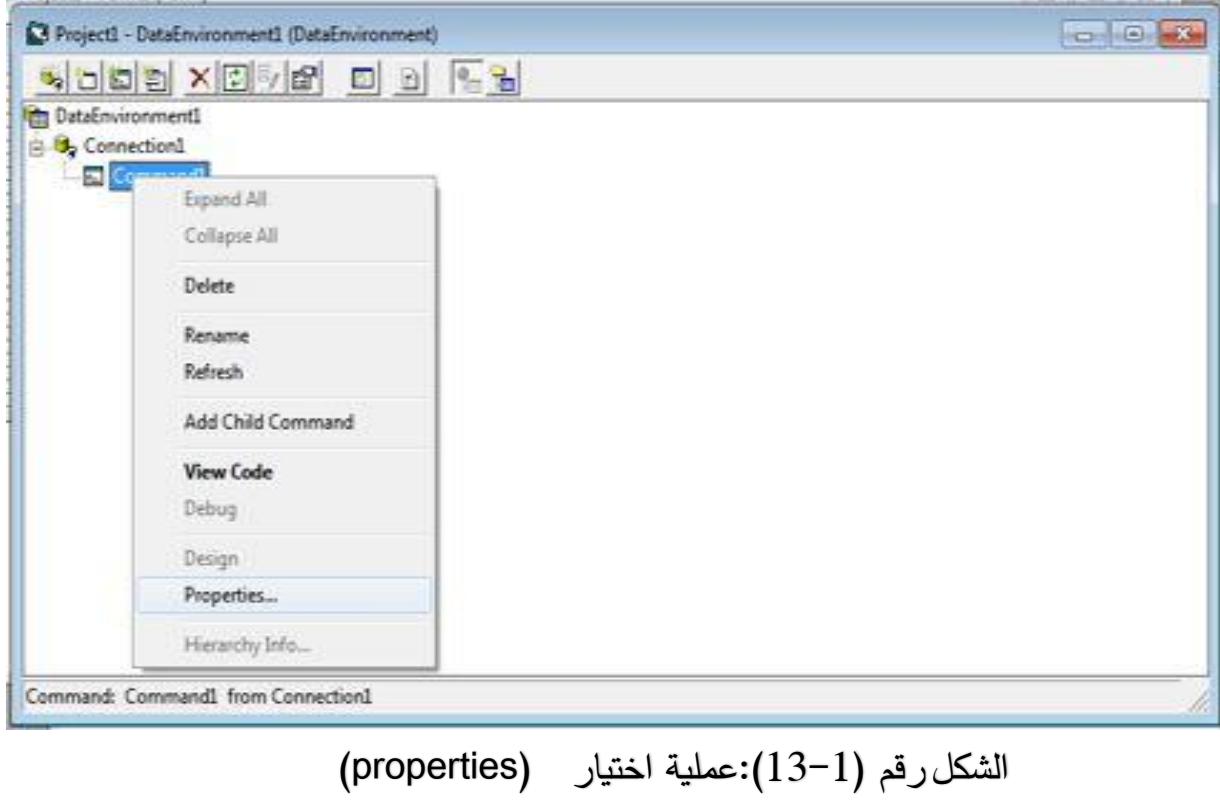




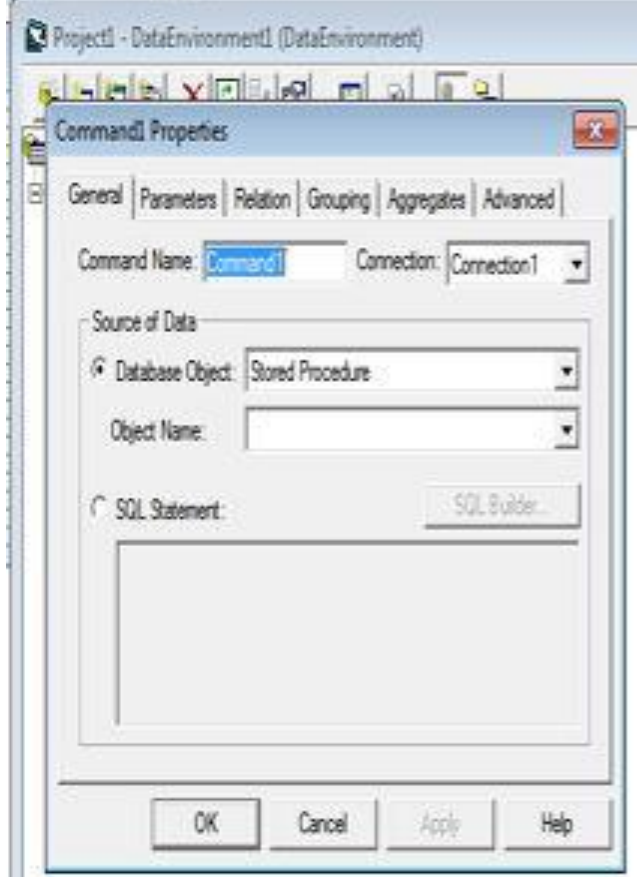

Commant Command frem Conection!

الشكل رقم (14-14):عملية اختيار (properties)

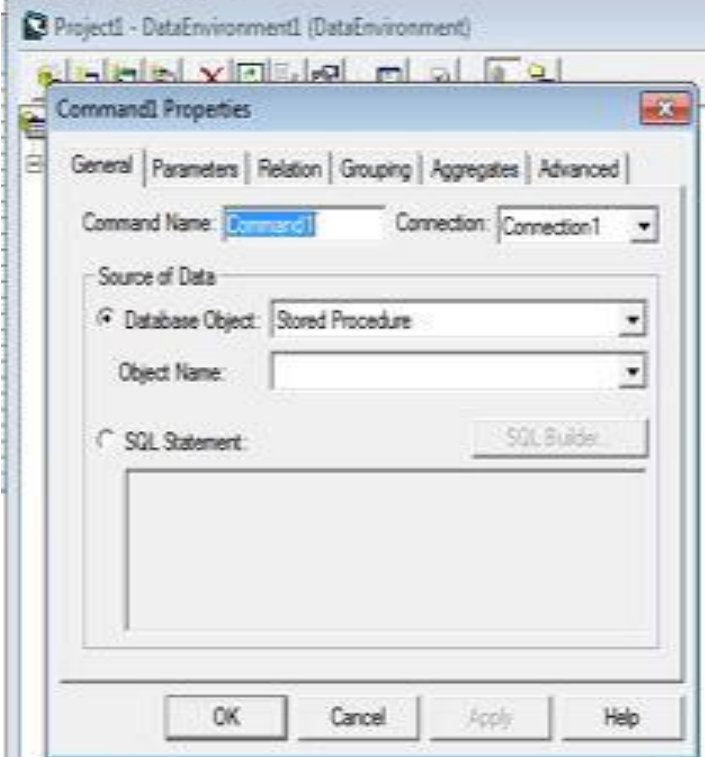

Command Command fism Connectionl

الشكل رقم (15-15):عملية اختيار (properties)

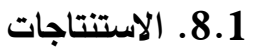

إن استخدام النظام الضريبي بالحاسوب أدى إلى زيادة البرعه في حساب الضريبة فضلًا عن

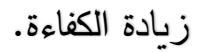


• حقيق الأمنية وذلك لاستخدامها عن طريق الموظف المخول بدلًا من ملئها عن طريق أشخاص

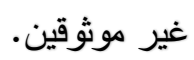

$$
\begin{aligned}
& \text { • زيادة سوعة البحث عن اسم الموظف المطلوب. } \\
& \text { • طباعة التقزير بسهولة عن الموظف. }
\end{aligned}
$$

• حفظ بيانات الموظفين في الحاسوب أسهل من الحفظ اليوي في استملات؛ لأنها معرضة

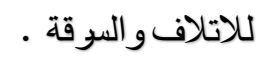

9.1

$$
\text { • عمل برنامج لحساب الرواتب فضلًا عن الضويبةوالتقاعد. }
$$

• •طوير النظام الضويبي للفودوجعله نظامًا ضويبًا عامًا لكافة المجتمع.

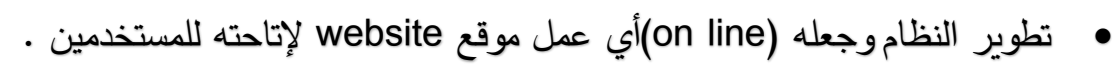

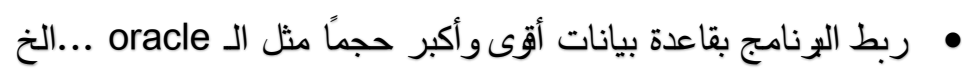


|لمصادر

م.م. قاسم محمد عبداله البعاج ،" اثر فاعلية نظم المعلومات الحاسبية في عملية التحاسب

الضريبي دراسة تطبيقية في الهيئة العامة للضرائب فرع الديوانية"،مجلة كلية الإدارة والاقتصاد ،

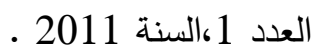

نسيبة عبدالرحمن محمد ،داليا محمود محمد ،شيماء فتحي حمد ،"حوسبة نظام تحصيل ضريبة

الدخل الثخصي "دراسة حالة في ديوان الضرائب ، جامعة السودان للعلوم والتكنولوجيا ،كلية

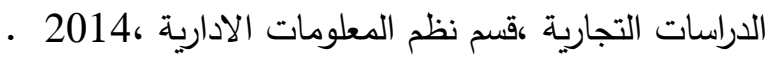

[3] Ramez Elmasri، Shamkant B. N avathe،2003,"Fundamentals of Database Systems".

[4] Abiteboul, S; Hull,R.; and Vianu,2003," Foundations of Databases" .

[5] Raghu Ramakrishnan 'Johannes Gehrke 'Jeff Derstadt, Scott Selikoff, and Lin Zhu،" DATABASE MANAGEMENT SYSTEMS SOLUTIONS MANUAL THIRD EDITION" ‘On-Line Learning Center Available at www.mhhe.com/ramakrishnan and at www.cs.wisc.edu/ dbbook. 